\title{
On Normal q-Ary Codes in Rosenbloom-Tsfasman Metric
}

\author{
R. S. Selvaraj and Venkatrajam Marka \\ Department of Mathematics, National Institute of Technology Warangal, Andhra Pradesh 506004, India \\ Correspondence should be addressed to R. S. Selvaraj; rsselva@nitw.ac.in
}

Received 10 February 2014; Accepted 17 March 2014; Published 2 April 2014

Academic Editors: A. Cossidente, E. Manstavicius, and S. Richter

Copyright (C) 2014 R. S. Selvaraj and V. Marka. This is an open access article distributed under the Creative Commons Attribution License, which permits unrestricted use, distribution, and reproduction in any medium, provided the original work is properly cited.

The notion of normality of codes in Hamming metric is extended to the codes in Rosenbloom-Tsfasman metric (RT-metric, in short). Using concepts of partition number and $l$-cell of codes in RT-metric, we establish results on covering radius and normality of $q$-ary codes in this metric. We also examine the acceptability of various coordinate positions of $q$-ary codes in this metric. And thus, by exploring the feasibility of applying amalgamated direct sum method for construction of codes, we analyze the significance of normality in RT-metric.

\section{Introduction}

Covering properties of codes have unique significance in coding theory, and covering radius, one of the four fundamental parameters, of a code is important in several respects [1]. Considering the fact that it is a geometric property of codes that characterizes maximal error correcting capability in the case of minimum distance decoding, covering radius had been extensively studied by many researchers (see, e.g., $[2,3]$ and the literature therein) especially with respect to the conventional Hamming metric. In fact, it has evolved into a subject in its own right mainly because of its practical applicability in areas such as data compression, testing, and write-once memories and also because of the mathematical beauty that it possesses. More on covering radius can be found in the monograph compiled by Cohen et al. [4]. In order to improve upon the bounds on covering radius, various construction techniques that use two or more known codes to construct a new code were proposed over the last few decades. One such method is direct sum construction which is a basic yet useful construction method. To improve upon the bounds related to covering radius of codes obtained using this method, the notion of normality which facilitates a construction technique known as amalgamated direct sum (ADS) was introduced for binary linear codes by Graham and Sloane in [5]. The same concepts were extended to binary nonlinear codes by Cohen et al. in [6]. Later, Lobstein and van Wee generalized these results to $q$-ary codes [7].

In the present paper, we extend the notion of normality to codes in Rosenbloom-Tsfasman metric (RT-metric, in short). The present work is a generalization of our work on binary codes [8] to $q$-ary case (though the discussed results hold good for codes over any alphabet of size $q$, for simplicity, we have taken it to be the finite field $\mathbb{F}_{q}$ ). RTmetric was introduced by Rosenbloom and Tsfasman in [9] and independently by Skriganov in [10] and is more adequate than Hamming metric in dealing with channels in which errors have a tendency to occur with a periodic spikewise perturbation of period $s \in \mathbb{N}$. As a generalization of the classical Hamming metric with rich mathematical beauty and being advantageous over Hamming metric in dealing with certain channels $[9,10]$, this metric attracted the attention of coding theorists over the last two decades [11-13]. The covering problem in RT-metric was first dealt with by Yildiz et al. for RT-spaces over Galois Rings [14].

The organization of the present paper is as follows. In Section 2, some basic definitions and notations that are used in this paper are presented. In Section 3, we have studied the covering radius of $q$-ary codes in this metric by introducing two new tools, namely, partition number and $l$-cell which greatly reduce the difficulty in determining the covering radius. In Section 4, we investigate the normality of $q$-ary 
codes in RT-metric. In this section, we also discuss the possibilities of extending the direct sum and amalgamated direct sum (ADS) constructions from Hamming metric to RT-metric. And, finally, Section 5 provides the conclusions of this paper.

\section{Definitions and Notations}

For $x=\left(x_{1}, x_{2}, \ldots, x_{s}\right) \in \mathbb{F}_{q}^{s}$, where $\mathbb{F}_{q}=\mathrm{GF}(q)$ is a finite field of $q$ elements, we define the $\rho$-weight (RT-weight) of $x$ to be

$$
w t_{\rho}(x)=\max \left\{i \mid x_{i} \neq 0,1 \leq i \leq s\right\} .
$$

The RT-distance or $\rho$-distance between $x=\left(x_{1}, x_{2}, \ldots, x_{s}\right)$ and $y=\left(y_{1}, y_{2}, \ldots, y_{s}\right) \in \mathbb{F}_{q}^{s}$ can be defined by $d_{\rho}(x, y)=$ $w t_{\rho}(x-y)$. The subsets of the space equipped with this metric are called RT-metric codes over $\mathbb{F}_{q}$ (or $q$-ary RT-metric codes) and the subspaces are called linear RT-metric codes over $\mathbb{F}_{q}$. The value $d_{\text {min }}=\min \left\{d_{\rho}(x, y): x, y \in C\right\}$ is called the minimum $\rho$-distance of the code $C$. The maximum distance of any word in the ambient space from an RT-metric code is called the covering radius of the code. A code in which each nonzero codeword is of the same weight $w_{\rho}$ is said to be a constant weight code. A $q$-ary code of length $s$, cardinality $K$, and minimum $\rho$-distance $d_{\rho}$ is said to be a maximum distance separable code (an MDS code, in short) if $K=q^{\left(s-d_{\rho}+1\right)}$.

Throughout this paper, unless otherwise specified, by a code we mean a $q$-ary RT-metric code and by distance we mean RT-distance. Moreover, $\left[s, k, d_{\rho}\right]_{q} R$ denotes a $q$-ary linear code of length $s$, dimension $k$, minimum distance $d_{\rho}$, and covering radius $R,\left(s, K, d_{\rho}\right)_{q} R$ denotes a $q$-ary code with cardinality $K$, and $t_{q}[s, k]$ denotes the minimum covering radius that a $q$-ary linear code of length $s$ and dimension $k$ can possess.

Definition 1 (direct sum of two codes). Let $C_{1}$ and $C_{2}$ be codes with parameters $\left(s_{1}, K_{1}\right)_{q}$ and $\left(s_{2}, K_{2}\right)_{q}$, respectively. Then, the direct sum of $C_{1}$ and $C_{2}$, denoted by $C_{1} \bigoplus C_{2}$, is defined as

$$
C_{1} \bigoplus C_{2}=\left\{\left(c_{1}, c_{2}\right) \mid c_{1} \in C_{1}, c_{2} \in C_{2}\right\} \text {, }
$$

and is a code of length $s_{1}+s_{2}$ and cardinality $K_{1} K_{2}$.

Definition 2 ( $q$-ary normal codes). Let $C$ be a $q$-ary RT-metric code of length $s$, cardinality $K$, and covering radius $R$, and, also, for each $a \in \mathbb{F}_{q}$, let $C_{a}^{(i)}$ denote the set of codewords in which the $i$ th coordinate is $a$. Then, the norm of $C$ with respect to the $i$ th coordinate is defined as

$$
N^{(i)}=\max _{x \in \mathbb{F}_{q}^{s}}\left\{\sum_{a \in \mathbb{F}_{q}} d_{\rho}\left(x, C_{a}^{(i)}\right)\right\}
$$

where

$$
d_{\rho}\left(x, C_{a}^{(i)}\right)= \begin{cases}\min \left\{d_{\rho}(x, y) \mid y \in C_{a}^{(i)}\right\} & \text { if } C_{a}^{(i)} \neq \emptyset \\ s & \text { if } C_{a}^{(i)}=\emptyset\end{cases}
$$

Now, $N=\min _{i} N^{(i)}$ is called the norm of $C$ and the coordinates $i$ for which $N^{(i)}=N$ are said to be acceptable.
Finally, a code is said to be normal if its norm satisfies $N \leq$ $q R+q-1$. If the code $C$ is not clear from the context, we use the notations $N^{(i)}(C)$ and $N(C)$.

\section{Covering Radius of RT-Metric Codes over $\mathbb{F}_{q}$}

Definition 3 (partition number of a $q$-ary RT-metric code). Let $C$ be an $\left(s, K, d_{\rho}\right)_{q}$ code in RT-metric. The largest nonnegative integer $l$, for which each $q$-ary $l$-tuple can be assigned to at least one codeword whose last $l$ coordinates are actually that $l$-tuple, is called the partition number of the code $C$. The code with partition number $l$ can be partitioned into $q^{l}$ parts, each of which has the property that all its members have the same $q$-ary $l$-tuple as their last $l$ coordinates. A part obtained in the above fashion is called an $l$-cell of the code, if at least one field element is not present in the $(s-l)$ th coordinate of its member codewords. As the definition of partition number suggests, a code with partition number $l$ contains at least $q^{l}$ codewords.

If $C$ is an $\left[s, k, d_{\rho}\right]_{q}$ linear code, then each of the $q^{l}$ parts does have $q^{k-l}$ codewords in it and so does each $l$-cell by its definition. If $C_{1}$ is an $l$-cell of the linear code $C$, then $x+C_{1}$ for $x \in C \backslash C_{1}$ will also be an $l$-cell different from $C_{1}$. Thus, for linear codes, if there is one $l$-cell, then there will be $q^{l}$ such $l$-cells.

Now, we will observe that covering radius of a code can be determined using the concepts of partition number and $l$-cell. The definition of partition number serves as a tool in determining the covering radius of an RT-metric code, as shown by the following theorem.

Theorem 4. Let $C$ be an $\left(s, K, d_{\rho}\right)_{q} R$ code in RT-metric. Then, the partition number of $C$ is $l$ if and only if its covering radius is $s-l$.

Proof. First, let the partition number of the code be $l$. Partition the code such that codewords in each part contain a unique $q$-ary $(l+1)$-tuple as their last $l+1$ coordinates. Associate each part with the respective $(l+1)$-tuple. Since the partition number of the code is $l$, the number of such parts will be less than $q^{l+1}$. Choose an $x \in \mathbb{F}_{q}^{s}$, whose last $l+1$ coordinates constitute the $(l+1)$-tuple that does not have a part associated with it. Such an $x$ will be at distance $s-l$ from the code, which is the maximum distance that a word can actually be from the code $C$. Hence, the covering radius is $s-l$.

Conversely, let the covering radius be $R=s-l$. By the definition of covering radius, any word must be at distance at most $s-l$ from the code, which means that, for each word, there is at least one codeword which agrees with the word in the last $s-(s-l)=l$ coordinate positions. This implies that the partition number of the code is greater than or equal to $l$. Let us assume that the partition number of the code is $m>$ $l$. Then, to each $q$-ary $m$-tuple, we can associate a codeword whose last $m$ coordinates coincide with that $m$-tuple. Thus, each word in $\mathbb{F}_{q}^{s}$ will be at distance at most $s-m$, contradicting the fact that covering radius of $C$ is $s-l$. Hence, the partition number of the code $C$ is $l$. 
Now, the above theorem can be restated in terms of $l$ cell in the following manner, as the definition of the $l$-cell suggests.

Corollary 5. Let $C$ be an RT-metric code of length sover $\mathbb{F}_{q}$. Then, the covering radius of $C$ is $s-l$ if and only if $\exists$ an l-cell of the code $C$.

Proposition 6. Let $C_{1}$ and $C_{2}$ be any two RT-metric codes over $\mathbb{F}_{q}$ with parameters $\left(s_{1}, K_{1}, d_{1}\right)_{q} R_{1}$ and $\left(s_{2}, K_{2}, d_{2}\right)_{q} R_{2}$, respectively. Then, their direct sum $C=C_{1} \oplus C_{2}$ is an $\left(s_{1}+\right.$ $\left.s_{2}, K_{1} K_{2}, d\right)_{q} R$ code with minimum distance $d=d_{1}$ and covering radius $R=s_{1}+R_{2}$, provided $C_{2} \neq \mathbb{F}_{q}^{s_{2}}$.

Proof. The direct sum of $C_{1}$ and $C_{2}$ is given by

$$
C_{1} \bigoplus C_{2}=\left\{\left(c_{1}, c_{2}\right) \mid c_{1} \in C_{1}, c_{2} \in C_{2}\right\} \text {. }
$$

Clearly, this is a code of length $s_{1}+s_{2}$ and cardinality $K_{1} K_{2}$. Now, since the minimum distance of $C_{1}$ is $d_{1}$, there exist two codewords $c_{1}$ and $c_{1}^{\prime}$ in $C_{1}$ such that $d_{\rho}\left(c_{1}, c_{1}^{\prime}\right)=d_{1}$. Then, the codewords $c=\left(c_{1}, c_{2}\right)$ and $c^{\prime}=\left(c_{1}^{\prime}, c_{2}\right)$ for some $c_{2} \in C_{2}$ will also be at distance $d_{1}$ which is minimum among all the codewords of $C$. From Theorem 4 and Definition 3, it is clear that the covering radius of a code in RT-metric depends on the partition number of the code and that the process of partitioning starts with the right most coordinate. Therefore, by Theorem 4 , unless $C_{2}$ is the space $\mathbb{F}_{q}^{s_{2}}$, the partition number of $C$ must be equal to that of $C_{2}$ which is $s_{2}-R_{2}$ and, hence, the covering radius of $C$ is $s_{1}+s_{2}-\left(s_{2}-R_{2}\right)=s_{1}+R_{2}$.

Remark 7. If $C_{2}=\mathbb{F}_{q}^{s_{2}}$, then the covering radius of the code $C=C_{1} \bigoplus C_{2}$ is $R=R_{1}$.

\section{Normality of Codes in RT-Metric}

Proposition 8. If C is an $\left(s, K, d_{\rho}\right)_{q} R R T$-metric code over $\mathbb{F}_{q}$, then $N(C) \geq q R$.

Proof. The proof follows directly from the definition of covering radius and that of norm of a code.

Theorem 9. Any q-ary RT-metric code of length s and covering radius $s$ is normal and all the coordinates are acceptable.

Proof. As $R=s$, the partition number is 0 which means that there exists an $\alpha \in \mathbb{F}_{q}$ which is not present as the last coordinate of any codeword in $C$. If $x=\left(x_{1}, x_{2}, \ldots, x_{s}\right) \in \mathbb{F}_{q}^{s}$ is such that $x_{s}=\alpha$, then $d_{\rho}\left(x, C_{a}^{(i)}\right)=s$ for each $a \in \mathbb{F}_{q}$ and for any $i \in\{1,2, \ldots, s\}$. Thus, $N^{(i)}=q s<q s+q-1=$ $q R+(q-1)$. Hence, the code is normal and all the coordinates are acceptable.

Lemma 10. Let $C$ be any RT-metric code with length $s$ and covering radius $R<s$; then

$$
N^{(i)}=(q-1) i+R,
$$

for each $i \in\{R+1, R+2, \ldots, s\}$.
Proof. For a coordinate position $i \in\{R+1, R+2, \ldots, s\}$, the norm is given by

$$
N^{(i)}=\max _{x \in \mathbb{E}_{q}^{s}}\left\{\sum_{a \in \mathbb{F}_{q}} d_{\rho}\left(x, C_{a}^{(i)}\right)\right\} .
$$

As the covering radius of $C$ is $R$, it has partition number $s-R$. So, the definition of partition number implies that each codeword can be associated with a unique $(s-R)$-tuple which actually consists of the last $(s-R)$ coordinates of that codeword and, also, that there exists at least one $(s-R+1)$ tuple which is not the same as the last $s-R+1$ coordinates of any of the codewords. Now, when we partition the code into $q$ parts $C_{a}^{(i)}$, for $a \in \mathbb{F}_{q}$, we can also partition the set of all $(s-R)$-tuples into $q$ parts corresponding to the associated codewords in $C_{a}^{(i)}$. If we choose a word $x \in \mathbb{F}_{q}^{s}$ whose last $s-R+1$ coordinates do not match with the last $s-R+1$ coordinates of any of the codewords, then, for this word, $\sum_{a \in \mathbb{E}_{q}} d_{\rho}\left(x, C_{a}^{(i)}\right)=(q-1) i+R$ which is the maximum value that a word can give. Hence, the proof holds.

Theorem 11. Let $C$ be any RT-metric code of length $s$ with covering radius $R<s$. Then, the coordinates $i \in\{R+2, R+$ $3, \ldots, s\}$ are not acceptable.

Proof. The minimum norm is

$$
N \leq N^{(i)}, \quad \forall i \in\{1,2, \ldots, s\} .
$$

By Lemma $10, N^{(i)}=(q-1) i+R$, for all $i \geq R+1$, which implies

$$
N^{(R+1)}<N^{(R+2)}<N^{(R+3)}<\cdots<N^{(s)} .
$$

From (8) and (9), one can conclude that

$$
N<N^{(i)}, \quad \forall i \in\{R+2, R+3, \ldots, s\} .
$$

This completes the proof.

The above theorem is not sufficient to arrive at a decision on the acceptability of the sth coordinate, when the code has covering radius $R=s-1$. This can be settled by the following theorem which says that no $q$-ary linear RT-metric code with dimension more than 1 and covering radius $s-1$ has acceptable last coordinate.

Theorem 12. Let $C$ be an $\left[s, k, d_{\rho}\right]_{q} R$ linear RT-metric code over $\mathbb{F}_{q}$ with $k>1$ and $R=s-1$. Then, the last coordinate is not acceptable.

Proof. By Lemma 10,

$$
\begin{aligned}
N^{(s)} & =(q-1) s+R \\
& =(q-1) s+s-1=q s-1 \\
& =q(s-1)+q-1=q R+(q-1)
\end{aligned}
$$

(since $R=s-1$ ). 
In order to prove this theorem, we must show that there exists at least one coordinate $j$ for which $N^{(j)}<N^{(s)}$. As the dimension of $C$ is $k$, there will be $k$ coordinate positions $i_{1}, i_{2}, \ldots, i_{k}$ such that $C_{a}^{\left(i_{t}\right)} \neq \emptyset$ for all $a \in \mathbb{F}_{q}$ and for each $t=1,2, \ldots, k$. One such coordinate position is $s$ (but not $s-1$, as the partition number is 1$)$. As $k>1$, there exists at least one more such coordinate position $j \in\left\{i_{1}, i_{2}, \ldots, i_{k}\right\}$ and $j \neq s$ wherein $C_{a}^{(j)} \neq \emptyset$ for all $a \in \mathbb{F}_{q}$, and so $N^{(j)} \leq q(s-1)<N^{(s)}$. This completes the proof.

But, when the code is either of dimension 1 or of constant weight, all the coordinates become acceptable as the following results suggest.

Proposition 13. Let $C$ be an $[s, 1]_{q}$ linear RT-metric code. Then, $C$ is normal and all the coordinates are acceptable.

Proof. It can easily be seen that the covering radius of $C$ is either $s$ or $s-1$. If it is $s$, the result follows by Theorem 9. If it is $s-1$, there exists a 1 -cell. Since $C$ is of dimension 1 , there exist $q$ such 1 -cells. By Lemma $10, N^{(s)}=q s-1$. Now for each $i \epsilon$ $\{1,2, \ldots, s-1\}$, either $C_{0}^{(i)}=C$ or $\left|C_{0}^{(i)}\right|=1$. In the former case, $C_{a}^{(i)}=\emptyset$ for all $a \neq 0$ and, in the latter case, $\left|C_{a}^{(i)}\right|=1$ for all $a \in$ $\mathbb{F}_{q}$. In any case, $N^{(i)}=q s-1$. This proves the result stated.

Proposition 14. Let $C$ be a constant weight code of length $s$ over $\mathbb{F}_{q}$. Then, $C$ is normal and all the coordinates are acceptable.

Proof. It is easy to see that the covering radius $R$ of the constant weight code $C$ is either $s$ or $s-1$, as it can have partition number of either 0 or 1 . If $R=s$, then, by Lemma 10, it is done. If $R=s-1$, then $C_{a}^{(s)} \neq \emptyset$ for any $a \in \mathbb{F}_{q}$. Then, for any $i, \overline{0} \in C_{0}^{(i)}$ and $\left|C_{0}^{(i)}\right| \geq 1$. For an $x \in \mathbb{F}_{q}^{s}$ with $x_{s}=0$ and $x_{s-1} \neq 0, N^{(i)}$ equals $q s-1$. Hence, the result follows.

Remark 15. If $C=\mathbb{F}_{q}^{s}$, then its partition number is $s$ and so its covering radius $R$ is 0 . By (3), for any $i, N^{(i)}=(q-1) i+0=$ $(q-1) i$. And so, $N^{(1)}<N^{(2)}<\cdots<N^{(s)}$ and $N^{(1)}=q-1=$ $q R+q-1$. Thus, the ambient space $\mathbb{F}_{q}^{s}$ is normal with the first coordinate being the only acceptable coordinate.

\subsection{Normality and Direct Sum Construction}

Proposition 16. Let $C_{1}$ and $C_{2}$ be $\left(s_{1}, K_{1}, d_{1}\right)_{q} R_{1}$ and $\left(s_{2}, K_{2}, d_{2}\right)_{q} R_{2}$ RT-metric codes, respectively, such that $R_{2} \neq 0$. Then,

(i) the direct sum $C=C_{1} \oplus C_{2}$ is normal;

(ii) all the coordinates corresponding to $C_{1}$ are acceptable for $C$;

(iii) the coordinate ifor $s_{1}+1 \leq i \leq s_{2}$ is acceptable for $C$ only if the norm of $C_{2}$ with respect to the coordinate $i-s_{1}$ is equal to $q R_{2}$.

Proof. Let $C_{1}$ and $C_{2}$ be any $\left(s_{1}, K_{1}, d_{1}\right)_{q} R_{1}$ and $\left(s_{2}\right.$, $\left.K_{2}, d_{2}\right)_{q} R_{2} q$-ary RT-metric codes, respectively. Then, by
Proposition 6, their direct sum $C=C_{1} \bigoplus C_{2}$ is an $\left(s, K_{1} K_{2}\right.$, $\left.d_{1}\right)_{q} R$ code with length $s=s_{1}+s_{2}$ and covering radius $R=s_{1}+R_{2}$. Now, for $i \in\left\{1,2, \ldots, s_{1}\right\}$, by (3), we have

$$
N^{(i)}(C)=q\left(s_{1}+R_{2}\right) \text {. }
$$

Now, in order to determine the norm $N(C)$, we have to find $N^{(j)}(C)$ for each $j \in\left\{s_{1}+1, s_{1}+2, \ldots, s_{1}+s_{2}\right\}$, the coordinate positions corresponding to $C_{2}$. But, we know by Proposition 8 that $N\left(C_{2}\right) \geq q R_{2}$ which implies $N^{(j)}(C) \geq q\left(s_{1}+R_{2}\right)$. Hence, $N(C)=q\left(s_{1}+R_{2}\right)=q R<q R+q-1$. Thus, $C$ is normal and all the coordinate positions corresponding to $C_{1}$ are acceptable and the coordinate position $j \in\left\{s_{1}+1, s_{1}+2, \ldots, s_{1}+s_{2}\right\}$ corresponding to $C_{2}$ is acceptable if $N^{\left(j-s_{1}\right)}\left(C_{2}\right)=q R_{2}$.

4.2. Normality and Amalgamated Direct Sum Construction. The amalgamated direct sum of two codes is defined as follows.

Definition 17. Let $C_{1}$ be an $\left[s_{1}, k_{1}\right]_{q} R_{1}$ normal code with the last coordinate being acceptable and let $C_{2}$ be an $\left[s_{2}, k_{2}\right]_{q} R_{2}$ normal code with the first coordinate being acceptable. Then, their amalgamated direct sum (or shortly ADS), denoted by $C_{1} \oplus C_{2}$, is an $\left[s_{1}+s_{2}-1, k_{1}+k_{2}-1\right]_{q}\left(s_{1}+R_{2}-1\right)$ code and is given by

$$
C_{1} \bigoplus C_{2}=\left\{\left(c_{1}|u| c_{2}\right):\left(c_{1} \mid u\right) \in C_{1}, \quad\left(u \mid c_{2}\right) \in C_{2}\right\} .
$$

Here, in this definition, we consider those codes $C_{1}$ and $C_{2}$ which are normal, respectively, with the last coordinate and first coordinate being acceptable such that the parts $C_{1_{a}}^{(s)}$ and $C_{2_{a}}^{(1)}$ are nonempty for all $a \in \mathbb{F}_{q}$.

As Theorem 12 shows the nonexistence of linear codes of dimension more than 1 whose last coordinate is acceptable, the significance of amalgamated direct sum and hence that of normality in RT-metric are less as far as the resulting covering radius is concerned. But, since the one-dimensional codes are normal and all the coordinates are acceptable, one can only use this ADS construction method to combine a 1-dimensional code with any other linear code, which may be helpful in the construction of MDS codes as seen in the following result.

Theorem 18. Let $C_{1}$ be an $\left[s_{1}, 1\right]_{q}$ MDS code with covering radius $t_{q}\left[s_{1}, 1\right]$ (which is normal with last coordinate being acceptable) and let $C_{2}$ be an $\left[s_{2}, k_{2}\right]_{q}$ MDS code with covering radius $t_{q}\left[s_{2}, k_{2}\right]$. Then, their amalgamated direct sum $C_{1} \oplus C_{2}$ is an $\left[s_{1}+s_{2}-1, k_{2}\right]_{q}$ MDS code with covering radius $t_{q}\left[s_{1}+\right.$ $\left.s_{2}-1, k_{2}\right]$.

Proof. From the definition of partition number and from Theorem 4 , it is easy to see that $t_{q}[s, k]=s-k$. So, $t_{q}\left[s_{1}, 1\right]=$ $s_{1}-1$ and $t_{q}\left[s_{2}, k_{2}\right]=s_{2}-k_{2}$. Now, as the dimension of $C_{1} \oplus C_{2}$ is $k_{2}$ (which is less than or equal to $s_{2}$ ), the covering radius of $C_{1} \oplus C_{2}$ depends only on the coordinates pertaining to the code $C_{2}$. But $C_{2}$ has covering radius $s_{2}-k_{2}$ and hence 
has partition number $k_{2}$. So, $C_{1} \oplus C_{2}$ also will have partition number $k_{2}$ and hence will have covering radius $s_{1}+s_{2}-1-k_{2}$ which is equal to $t_{q}\left[s_{1}+s_{2}-1, k_{2}\right]$, which implies that $C_{1} \oplus C_{2}$ is MDS. This completes the proof.

\section{Conclusion}

We discussed the normality of codes over $\mathbb{F}_{q}$ in RT-metric and determined its norm with respect to various coordinate positions. We also established that the last coordinate is not acceptable for any nontrivial code in this metric which makes the ADS construction less significant as far as the higher dimensional RT-metric codes are concerned.

\section{Conflict of Interests}

The authors declare that there is no conflict of interests regarding the publication of this paper.

\section{References}

[1] P. Delsarte, "Four fundamental parameters of a code and their combinatorial significance," Information and Control, vol. 23, no. 5, pp. 407-438, 1973.

[2] G. D. Cohen, M. G. Karpovsky, H. F. Mattson, and J. R. Schatz, "Covering radius-survey and recent results," IEEE Transactions on Information Theory, vol. 31, no. 3, pp. 328-343, 1985.

[3] G. D. Cohen, S. N. Litsyn, A. C. Lobstein, and H. F. Mattson Jr., "Covering radius 1985-1994," Applicable Algebra in Engineering, Communications and Computing, vol. 8, no. 3, pp. 173-239, 1997.

[4] G. D. Cohen, I. Honkala, S. Litsyn, and A. Lobstein, Covering Codes, Elsevier, North-Holland, The Netherlands, 1997.

[5] R. L. Graham and N. J. A. Sloane, "On the covering radius of codes," IEEE Transactions on Information Theory, vol. 31, no. 3, pp. 385-401, 1985.

[6] G. D. Cohen, A. C. Lobstein, and N. J. A. Sloane, "Further results on the covering radius of codes," IEEE Transactions on Information Theory, vol. 32, no. 6, pp. 680-694, 1986.

[7] A. C. Lobstein and G. J. M. van Wee, "On normal and subnormal $q$-ary codes," IEEE Transactions on Information Theory, vol. 35, no. 6, pp. 1291-1295, 1989.

[8] R. S. Selvaraj and V. Marka, "Normality of binary codes in Rosenbloom-Tsfasman metric," in Proceedings of the International Conference on Advances in Computing, Communications and Informatics (ICACCI '13), pp. 1370-1373, August 2013.

[9] M. Yu. Rosenbloom and M. A. Tsfasman, "Codes for the $m$-metric," Problems of Information Transmission, vol. 33, pp. 45-52, 1997.

[10] M. M. Skriganov, "Coding theory and uniform distributions," St. Petersburg Mathematical Journal, vol. 143, no. 2, pp. 301-337, 2002.

[11] I. Siap and M. Ozen, "The complete weight enumerator for codes over $M_{n \times s}(R)$ with respect to the Rosenbloom-Tsfasman metric," Applied Mathematics Letters, vol. 17, no. 1, pp. 65-69, 2004.

[12] S. T. Dougherty and K. Shiromoto, "Maximum distance codes in $\operatorname{Mat}_{n \times s}\left(\mathbb{Z}_{k}\right)$ with a non-hamming metric and uniform distributions," Designs, Codes, and Cryptography, vol. 33, no. 1, pp. 4561, 2004.
[13] S. Jain, "CT bursts-from classical to array coding," Discrete Mathematics, vol. 308, no. 9, pp. 1489-1499, 2008.

[14] B. Yildiz, I. Siap, T. Bilgin, and G. Yesilot, "The covering problem for finite rings with respect to the RT-metric," Applied Mathematics Letters, vol. 23, no. 9, pp. 988-992, 2010. 


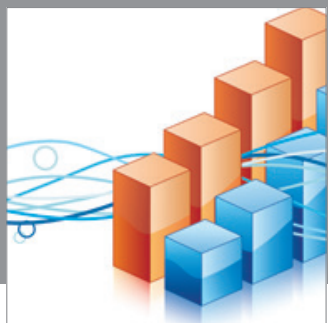

Advances in

Operations Research

mansans

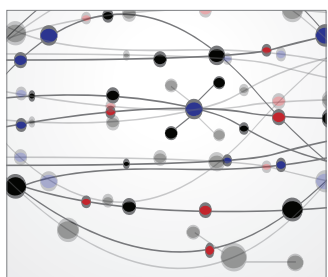

The Scientific World Journal
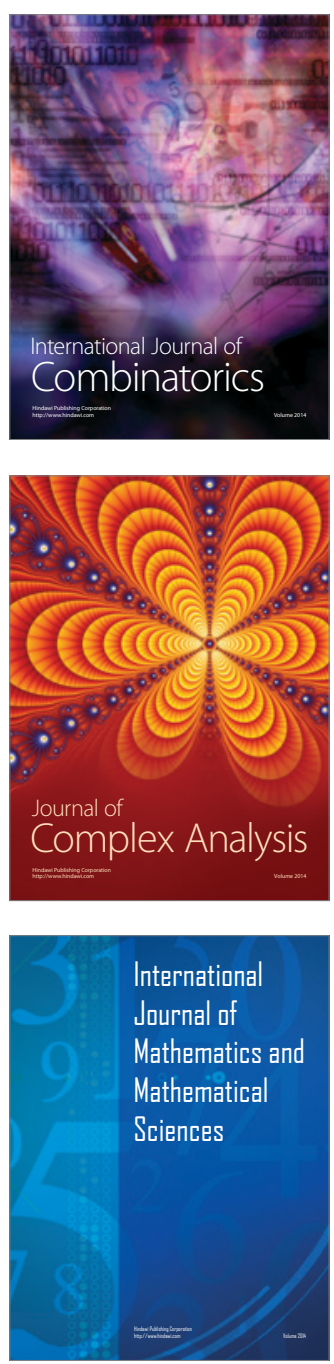
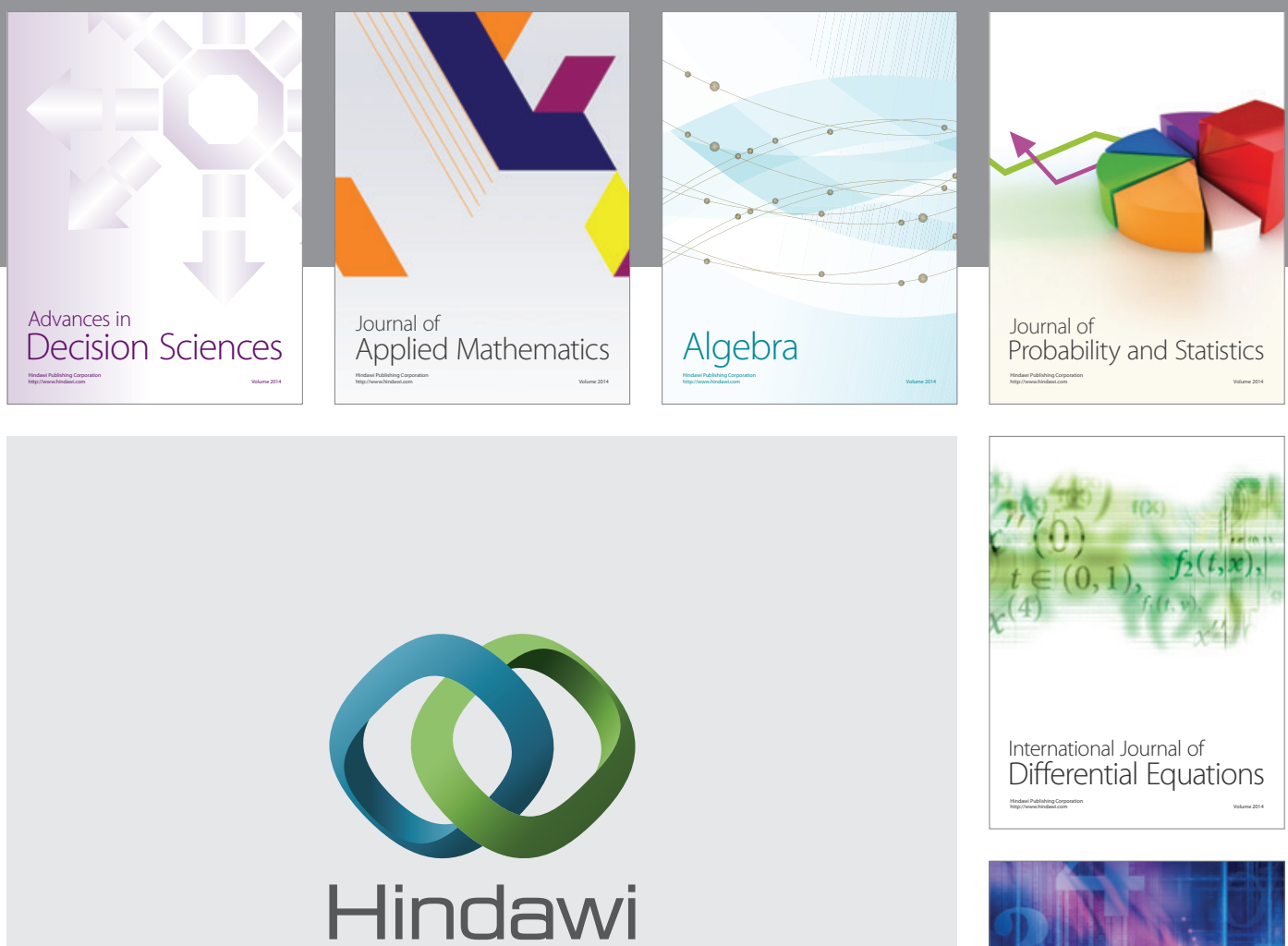

Submit your manuscripts at http://www.hindawi.com
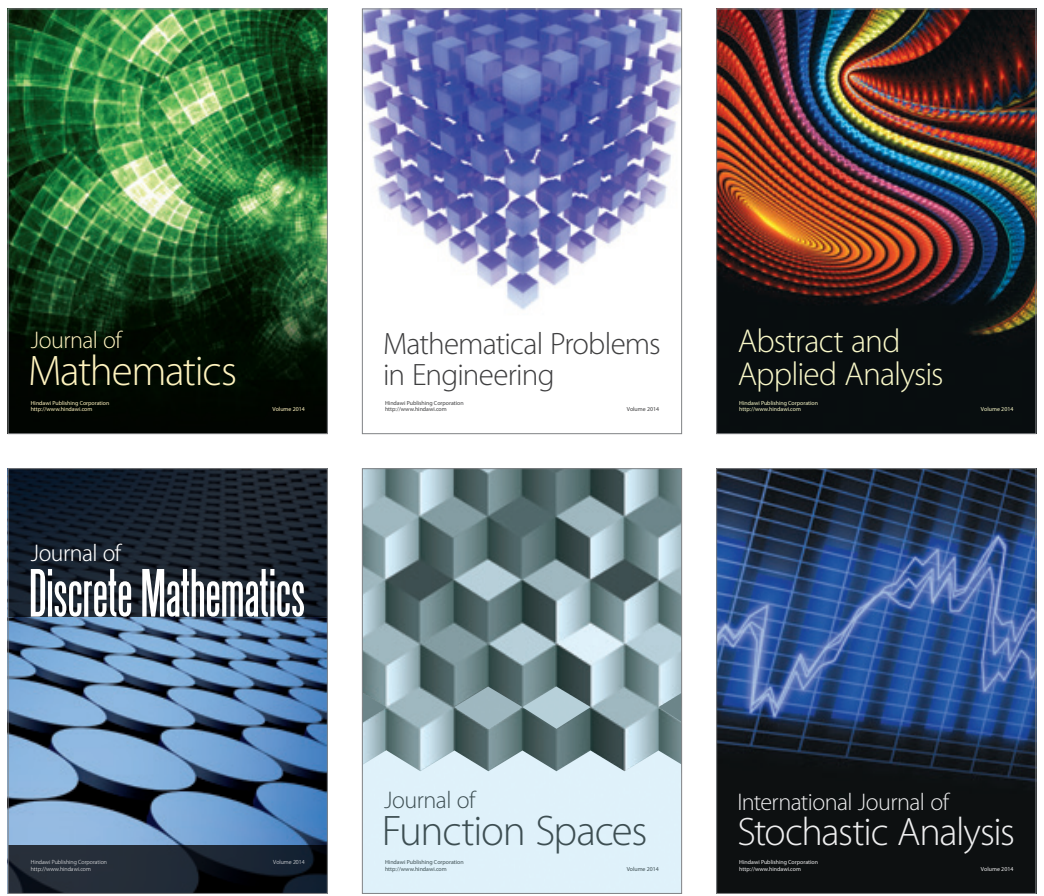

Journal of

Function Spaces

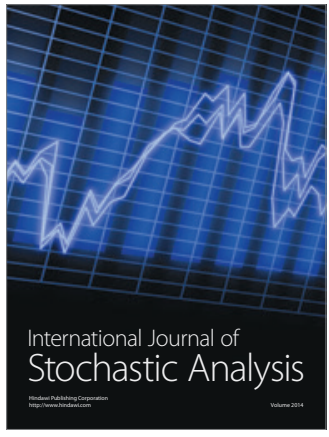

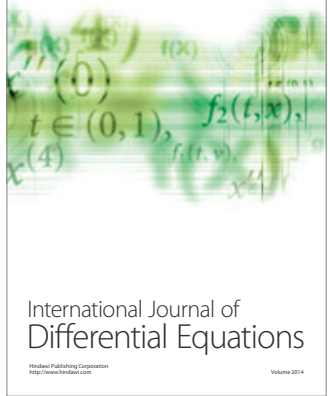
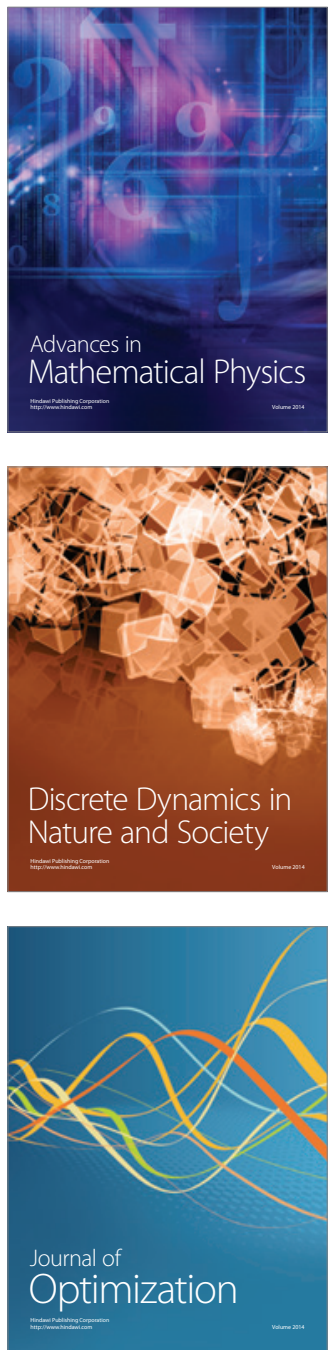